\title{
Uncommon Primary Synovial Chondromatosis Involving Only the Infrapatellar Fat Pad in an Elderly Patient
}

\author{
Dae-Hee Lee, $\mathrm{MD}^{1}$ and Tae-Wan Jeong, $\mathrm{MD}^{2}$ \\ ${ }^{1}$ Department of Orthopaedic Surgery, Samsung Medical Center, Sungkyunkwan University School of Medicine, Seoul; ${ }^{2}$ Department of Orthopedic Surgery, Korea \\ University Anam Hospital, Korea University College of Medicine, Seoul, Korea
}

\begin{abstract}
Primary synovial chondromatosis is a rare condition of idiopathic synovial chondrometaplasia and usually occurs during the third to fifth decades of life. Conversely, secondary synovial chondromatosis results from the growth of separated particles from articular cartilage or osteophytes in patients with joint diseases, such as degenerative osteoarthritis, and occurs mostly in elderly people. We describe here a 76-year-old male histopathologically confirmed as having primary synovial chondromatosis with no calcification of the infrapatellar fat pad (IFP) of the knee joint. To our knowledge, this is the first description of primary synovial chondromatosis of the knee joint confined to the IFP in a patient $>60$ years old.
\end{abstract}

Keywords: Knee, Chondromatosis, Synovial, Adipose

Synovial chondromatosis is a relatively uncommon monoarticular synovial proliferative disease, in which cartilaginous or osteocartilaginous metaplasia occurs within the synovial lining of joints, bursae, and/or tendon sheaths. Synovial chondromatosis can be classified as primary or secondary type. Primary synovial chondromatosis is a rare condition characterized by chondrometaplasia of the synovium of unknown cause and usually occurs during the third to fifth decades of life ${ }^{1,2)}$. In contrast, secondary synovial chondromatosis results from the growth of separated particles from articular cartilage or osteophytes in patients with joint diseases and synovial irritation, such as degenerative osteoarthritis, and occurs mostly in elderly people ${ }^{2)}$.

Received December 10, 2014; Revised March 20, 2015;

Accepted May 11, 2015

Correspondence to: Dae-Hee Lee, MD

Department of Orthopaedic Surgery, Samsung Medical Center,

Sungkyunkwan University School of Medicine, 81 Irwon-ro, Gangnamgu, Seoul 06351, Korea

Tel: +82-2-3410-3509, Fax: +82-2-3410-0061

E-mail: eoak22@empal.com

This is an Open Access article distributed under the terms of the Creative Commons Attribution Non-Commercial License (http://creativecommons.org/licenses/by-nc/4.0/) which permits unrestricted non-commercial use, distribution, and reproduction in any medium, provided the original work is properly cited.
The differential diagnosis of patients presenting with a solitary mass on the infrapatellar fat pad (IFP) is difficult, especially if plain radiographs show no evidence of calcification. Magnetic resonance imaging (MRI) is helpful in diagnosing patients with early phase synovial chondromatosis because it reveals uncalcified nodules and the degree of soft tissue involvement ${ }^{3,4}$.

We describe here a 76-year-old male histopathologically confirmed as having primary synovial chondromatosis, with no calcification, of the IFP of the knee joint. To our knowledge, this is the first description of primary synovial chondromatosis of the knee joint involving only the IFP in a patient $>60$ years old.

\section{Case Report}

A 76-year-old male presented with a six-month history of pain in his right knee joint. The pain occurred insidiously without any antecedent trauma. He exhibited no signs of meniscal tear, ligamentous injury, or limitation of motion, but had pain on terminal extension. He also complained of intermittent pain while walking, but he denied any pain at rest or constitutional symptoms.

An initial physical examination showed no specific swelling of the right knee joint. He experienced anterior knee pain on terminal extension with a limited knee extension of $10^{\circ}$. The patient 
showed neither ligamentous instability nor positive findings on mechanical tests.

Simple radiographs of the knee showed no definite osteoarthritic change on the tibiofemoal joint or a soft tissue density lesion, although small calcification was observed on the suprapatellar pouch (Fig. 1). T1-weighted MRI scans of the right knee joint showed a lobulated, well-defined mass in the IFP with intermediate signal intensity, whereas T2-weighted images showed homogeneous and relatively high signal intensity but no hypointense areas such as calcified nodules or hemosiderin deposits. Both T1 and $\mathrm{T} 2$ weighted images showed that the mass was bisected by

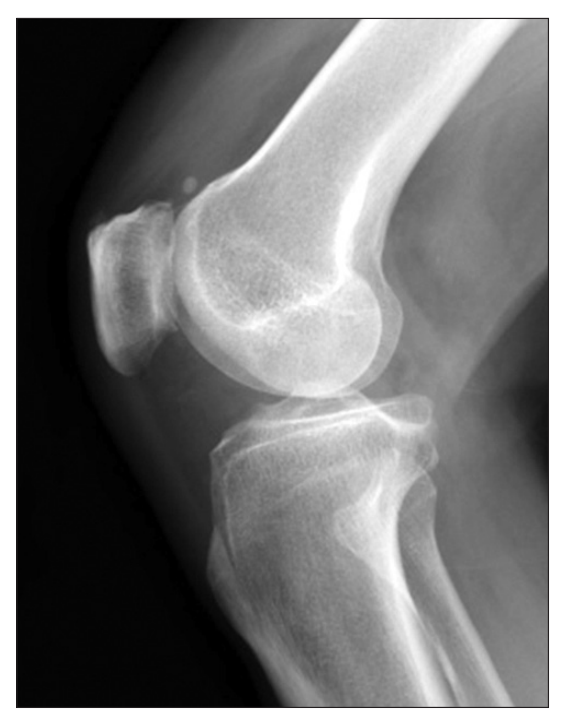

Fig. 1. Lateral radiograph of the right knee showing neither definite calcification nor a soft tissue density lesion. band-like septa of low signal intensity (Fig. 2).

The patient underwent arthroscopic excision. Conventional anteromedial and anterolateral portals were introduced to confirm the preoperative MRI findings. Far medial and far lateral portals were made to clearly visualize the mass and thoroughly remove it arthroscopically. The arthroscopic findings showed a whitish, ovoid-shaped mass buried in the IFP (Fig. 3A).

After debridement of some of the fat pad adjacent to the mass, a freely movable large-sized mass was exposed (Fig. 3B). The mass was completely excised with a punch and shaver and completely removed. No damage to the cruciate ligament was found but mild osteoarthritic changes, considering the old age, were shown, such as minimal meniscus tear and articular cartilage wear. Histopathological examination of the mass showed unevenly distributed plump chondrocytes with cellular atypia, some binucleated, within irregularly-shaped nests of cells. Neither zonal ring-like calcification nor enchondral ossification was observed (Fig. 4). These findings were consistent with a diagnosis of primary synovial chondromatosis. At 20-month follow-up, the patient was taking part in normal daily activities.

\section{Discussion}

The case presented here had unique characteristics in that the primary synovial chondromatosis occurred only in the IFP in a relatively elderly patient. Synovial chondromatosis in elderly patients is usually secondary, developing due to osteochondral fragments originating from osteoarthritis. In addition, the absence of indicators of calcification on plain radiographs made the

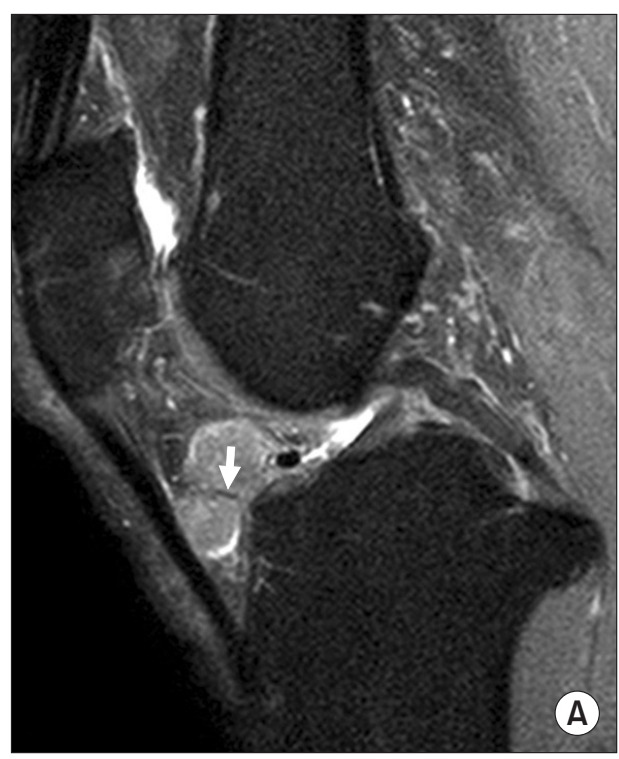

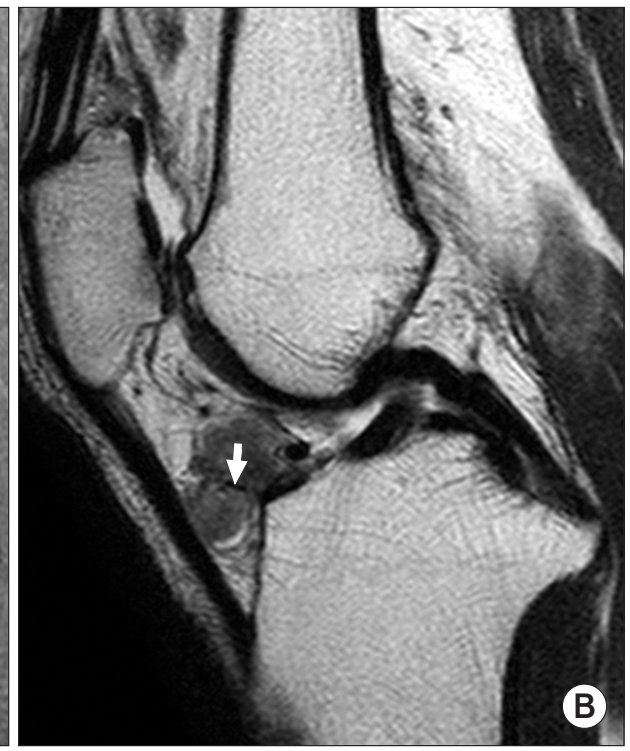

Fig. 2. Sagittal T1-weighted (A) and T2weighted (B) magnetic resonance imaging scans. A lobulated mass lesion of intermediate signal intensity was noted in the infrapatellar fat pad on the T1-weighted image (A) and of high signal intensity on the T2-weighted image (B). Both images showed a band-like component of low signal density (white arrows) suggesting that this lesion had fibrous septa, resulting in the diagnosis of primary, rather than secondary, chondromatosis. 

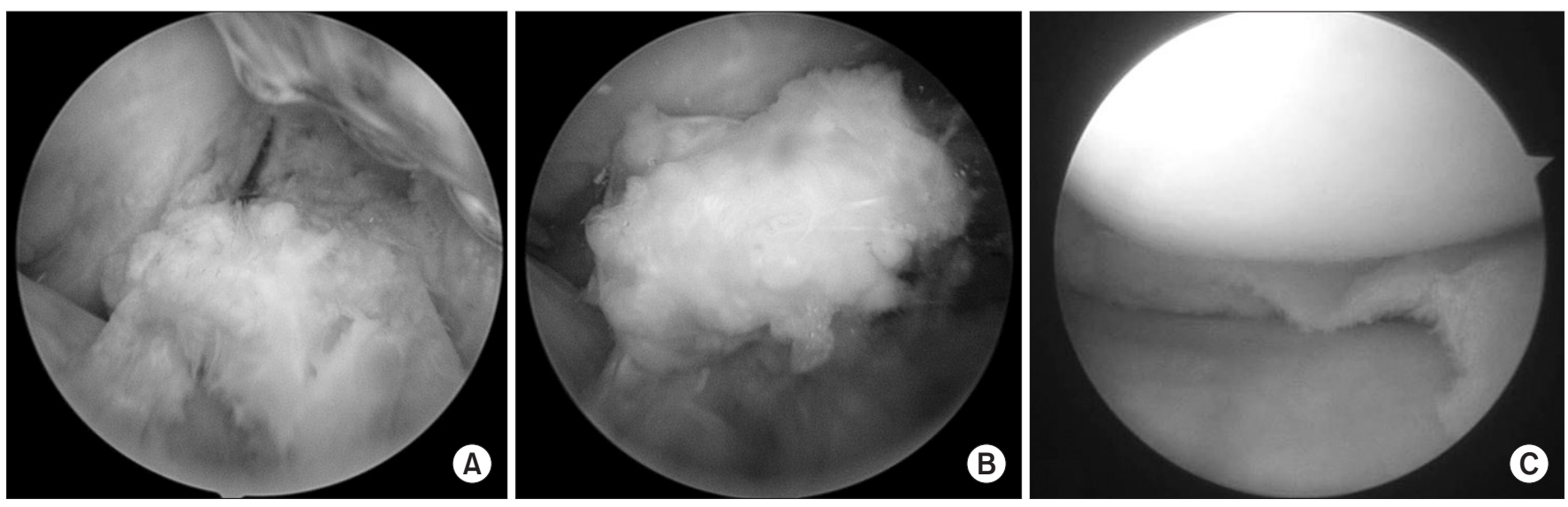

Fig. 3. (A) Arthroscopic view showing a whitish, ovoid-shaped loose body buried in the infrapatellar fat pad. (B) Debridement of the fat pad tissue surrounding the mass allowed the latter to move freely. (C) Articular chondral wear of the tibiofemoral joint was not severe considering the old age.
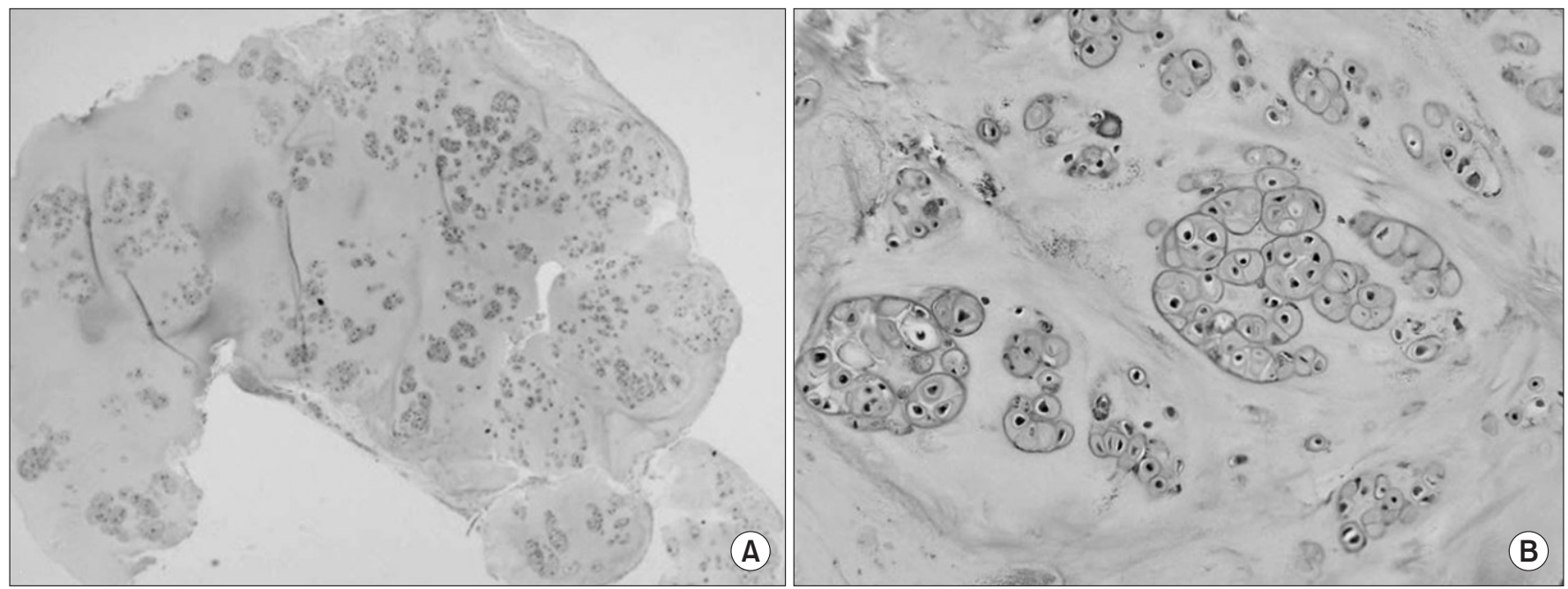

Fig. 4. (A) Histopathologic results showing uneven spatial distribution of disorganized growth of chondrocytes in a low power field (H\&E, $\times 100)$. (B) High power field image showing crowded, irregularly nested patterns of these chondrocytes with some cellular atypia, but no distinct calcific foci (H\&E, $\times 400)$.

differential diagnosis between primary synovial chondromatosis and localized pigmented villonodular synovitis (PVNS) difficult in the patient.

Although IFP involvement was observed in 6 of 12 patients (50\%) with synovial chondromatosis of the knee in the study by Helpert et al. ${ }^{5}$, chondromatosis was confined to the IFP in none of these patients, with all having another mass in the suprapatellar pouch and/or popliteal fossa ${ }^{5}$. Synovial chondromatosis involving the IFP was also found in a 14-year-old girl, but she also had a nodular lesion located posterior to the posterior cruciate ligament ${ }^{1}$.

Only one previous case of synovial chondromatosis involving only the IFP has been reported ${ }^{6}$. It was, however, relatively easy to diagnose because plain radiographs showed a calcified tissue mass in the anterior aspect of the knee, making MRI unnecessary. The diagnosis of the patient was confirmed by a computed tomography scan, which showed a calcified nodule extending into the IFP. In our patient, however, plain radiographs showed no specific abnormal findings around the IFP, such as calcification or bony erosion. These findings suggest that the lesion in our patient represented an earlier phase of synovial chondromatosis than that of the previously described case ${ }^{6}$, since calcification becomes more prominent as synovial chondromatosis progresses.

Synovial chondromatosis can be classified as primary or secondary type ${ }^{7}$, despite difficulties in the differential diagnosis of these types because of similar clinic-radiologic findings. The de- 
velopment of chondroid foci in the synovial membrane without identifiable etiological factors in young patients indicates primary synovial chondromatosis. In contrast, secondary chondromatosis occurs in relatively older patients and results from the growth of particles that have separated from the articular cartilage or osteophytes in patients with joint diseases, such as osteoarthritis ${ }^{8)}$. Histopathologically, primary synovial chondromatosis is not associated with any underlying disease, whereas secondary synovial chondromatosis is associated with degenerative joint diseases. Furthermore, the growth of cartilage cells is more disorganized in primary synovial chondromatosis, and the increased numbers of chondrocyte are often crowded and nested irregularly"). Cellular growth is more organized in secondary synovial chondromatosis, with cells often growing in concentric rings arranged in an orderly zone of transformation from fibrocartilage to hyalinetype cartilage through endochondral ossification to bone. Loose bodies originating from primary synovial chondrometaplasia can themselves initiate secondary synovial chondrometaplasia. For this reason, the histologic criteria we have presented constitute the only definitive way to differentiate the two conditions. The absence of enchondral ossification and the irregular distribution of closely packed plump chondrocytes clustering in nodules suggest that our patient likely had primary, rather than secondary, synovial chondromatosis, despite his elderly age.

It is especially difficult to differentiate a localized form of PVNS from synovial chondromatosis in patients with a non-cystic, noncalcified mass in the IFP. MRI can assist in the differential diagnosis of these patients. Plain radiographs of our patient showed no calcification or calcified soft tissue mass around the IFP. MRI showed that this mass had lobulated borders, with intermediate signal intensity on T1-weighted images and high signal intensity on T2-weighted images. Although there were no signal voids of calcified nodules, the mass showed hypointense septa in both sequences, corresponding to areas of fibrosis during the early phase of synovial chondromatosis ${ }^{4)}$. Moreover, the mass contained no hemosiderin deposits, a characteristic feature of PVNS, although these deposits are less notable in the localized than in the diffuse form of PVNS. Our MRI findings resulted in a preliminary diagnosis of synovial chondromatosis, rather than localized PVNS, prior to surgery.
In conclusion, our findings suggest that primary synovial chondromatosis may occur in elderly patients with anterior knee pain and MRI is very useful in diagnosing patients with early phase synovial chondromatosis without calcification.

\section{Conflict of Interest}

No potential conflict of interest relevant to this article was reported.

\section{References}

1. Chou PH, Huang TF, Lin SC, Chen YK, Chen TH. Synovial chondromatosis presented as knocking sensation of the knee in a 14-year-old girl. Arch Orthop Trauma Surg. 2007;127: 293-7.

2. Crotty JM, Monu JU, Pope TL Jr. Synovial osteochondromatosis. Radiol Clin North Am. 1996;34:327-42.

3. Kramer J, Recht M, Deely DM, Schweitzer M, Pathria MN, Gentili A, Greenway G, Resnick D. MR appearance of idiopathic synovial osteochondromatosis. J Comput Assist Tomogr. 1993;17:772-6.

4. Narvaez JA, Narvaez J, Aguilera C, De Lama E, Portabella F. MR imaging of synovial tumors and tumor-like lesions. Eur Radiol. 2001;11:2549-60.

5. Helpert C, Davies AM, Evans N, Grimer RJ. Differential diagnosis of tumours and tumour-like lesions of the infrapatellar (Hoffa's) fat pad: pictorial review with an emphasis on MR imaging. Eur Radiol. 2004;14:2337-46.

6. Osti L, Papalia R, Del Buono A, Denaro V, Maffulli N. Recurrence of synovial chondromatosis of the Hoffa's body. Knee Surg Sports Traumatol Arthrosc. 2009;17:1421-4.

7. Villacin AB, Brigham LN, Bullough PG. Primary and secondary synovial chondrometaplasia: histopathologic and clinicoradiologic differences. Hum Pathol. 1979;10:439-51.

8. Milgram JW. The classification of loose bodies in human joints. Clin Orthop Relat Res. 1977;(124):282-91.

9. Lin RC, Lue KH, Lin ZI, Lu KH. Primary synovial chondromatosis mimicking medial meniscal tear in a young man. Arthroscopy. 2006;22:803. 